\title{
A PROOF OF THE SLICING THEOREM FOR 2-SPHERES
}

BY NORMAN HOSAY

Communicated by R. H. Bing, April 16, 1968

1. Introduction. The purpose of this note is to answer in the affirmative the following question raised by Bing [3]: Is a 2-sphere $S$ in Euclidean 3-space $E^{3}$ tame if each horizontal cross-section of $S$ is either a point or a simple closed curve? (I have been informed by R. H. Bing that W. T. Eaton has independently proved a similar theorem.)

If $S$ is polyhedral that $S$ is flat is well known. A proof is sketched in [1] and more detailed proofs are given in [5] and [6]. However, the more general assertion posed by the above question was placed in doubt by an example due to Bing. In [3, p. 362] a nonpolyhedral, tame 2-sphere is constructed which satisfies the above condition but cannot be taken onto a round sphere by a level preserving homeomorphism of $E^{3}$.

The proof given here is not elementary in that it relies on two characterizations, due to Bing, [2] and [4], of tame 2-spheres in $E^{3}$. More will be said about these results when they are used.

I would like to thank R. H. Bing and I. Ferris for helpful comments.

We assume the usual metric on $E^{3}$ throughout this paper and in order to simplify the notation we let

$$
E_{t}=\left\{(x, y, z) \in E^{3} \mid z=t\right\} .
$$

2. THEOREM. Let $S^{2}$ be a 2-sphere in $E^{3}$ and $a<b$ real numbers such that for $a<t<b, S^{2} \cap E_{t}=J_{t}$ is a simple closed curve and $S^{2} \cap E_{t}=p_{t}$ is a point for $t$ equal to $a$ or $b$. Then $S^{2}$ is tame.

Proof. We first prove that $S^{2}$ is locally tame modulo $\left\{p_{a}, p_{b}\right\}$ by showing that the complementary domains of $S^{2}$ are locally simply connected at each point $p \in S^{2}-\left\{p_{a}, p_{b}\right\},[2]$. The two extreme points will be dealt with in the last step of the proof.

Given $p \in S^{2}-\left\{p_{a}, p_{b}\right\}$ let $U$ be a round open ball containing $p$ and small enough so that no $J_{t}$ lies entirely in $U$. Further assume that $U$ misses $E_{a}$ and $E_{b}$. Choose $V$ to be a round open ball containing $p$ such that $V \subset U$ and $V \cap S^{2}$ lies in a disk in $U \cap S^{2}$. Let $D$ be a 2-cell. We want to show that any map

$$
f: \operatorname{Bd} D \rightarrow V-S^{2}
$$


admits an extension

$$
g: D \rightarrow U-S^{2}
$$

We can assume that $f(\operatorname{Bd} D)$ is a polygonal simple closed curve such that no edge lies on a horizontal plane and that $f(\operatorname{Bd} D) \subset \operatorname{Int} S^{2} \cap V$. Since $V$ is simply connected there exists a piecewise linear map

$$
\hat{h}: D \rightarrow V
$$

such that

$$
\left.\hat{h}\right|_{\mathrm{Bd} D}=f \text { on } \mathrm{Bd} D \text {. }
$$

Let $C$ be the component of $D-\hat{h}^{-1}\left(\hat{h}(D) \cap S^{2}\right)$ containing Bd $D$. Since $\hat{h}(D) \cap S^{2}$ lies in a disk in $U \cap S^{2}$ there exists a map

$$
h: D \rightarrow U \cap \mathrm{Cl}\left(\operatorname{Int} S^{2}\right)
$$

such that

$$
\left.h\right|_{c}=\hat{h} \quad \text { on } C
$$

Also

$$
h_{\mid \mathrm{Bd} D}=f \text { on } \mathrm{Bd} D \text {. }
$$

Since $h$ is piecewise linear on a closed neighborhood in $D$ of $\operatorname{Bd} D$, for a fixed value $t$, only finitely many components of the set $h^{-1}\left(h(D) \cap E_{t}\right)$ will have the property that each contains more than one point of $\mathrm{Bd} D$. We call the components with this property $A_{1}^{\imath}, \cdots, A_{k(t)}^{\imath}$.

The remainder of the proof of this part of the theorem is divided in to four parts.

(A) In this part each set $h\left(A_{i}^{t}\right)$ is replaced by a certain singular finite graph. We begin by pushing $J_{t}$ slightly into Int $J_{t}$ on $E_{t}$. Since $S^{2} \cap E_{t}=J_{t}$, a simple closed curve, there exists a homeomorphism

$$
k: E_{t} \rightarrow E_{t}
$$

such that

$$
\left.k\right|_{h(\mathrm{Bd} D)} \cap E_{t}=1
$$

and

$$
k\left(h(D) \cap E_{t}\right) \subset U \cap \operatorname{Int} S^{2} \cap E_{t} .
$$

Let $O_{j} \subset U \cap I n t S^{2} \cap E_{t}$ be a connected open set about $k h\left(A_{j}^{t}\right)$ $(j=1, \cdots, k(t))$. Let $p$ be some point in $O_{j}$. We join each point $q \in h(\operatorname{Bd} D) \cap O_{j}$ to $p$ by an arc $[p q] \subset O_{j}$ and take $K_{j}^{\imath}$ to be the union 
of the finite number of arcs in $O_{j}$ obtained in this way. The arcs $[p q]$ may intersect each other badly.

(B) We now select a finite number of planes $E_{t_{1}}, \cdots, E_{t_{n}}$ so as to satisfy a certain homotopy condition. Let $K^{t}=K_{1}^{t} \cup \ldots \cup K_{k(t)}^{t}$. For each $t(a<t<b)$ let $\epsilon_{t}$ be a positive number such that $\epsilon_{t}<\rho\left(K^{t}, S^{2}\right)$ and $\epsilon_{t}<\rho\left(f(\operatorname{Bd} D), S^{2}\right)$. Let $V_{t^{\prime}}\left(K^{t}\right)$ be the vertical translate of $K^{t}$ to the horizontal plane $E_{i^{\prime}}$. Then $V_{t^{\prime}}\left(K^{t}\right) \cap S^{2}=\varnothing$ for $\left|t^{\prime}-t\right|<\epsilon_{t}$.

Let $e<f$ be real numbers such that $h(\mathrm{Bd} D) \subset \bigcup_{e \leq t \leq f} E_{t}$. We cover the interval $[e, f]$ by open intervals $W_{t}=\left(t-\epsilon_{t}, t+\epsilon_{t}\right), e \leqq t \leqq f$. Let $W_{t_{1}}, \cdots, W_{\iota_{n}}$ be a finite subcollection of the $W_{t}$ covering $[e, f]$ indexed so that $t_{1}<t_{2}<\cdots<t_{n}$. Let $t_{i}$ be a number in $W_{t_{i}} \cap W_{t_{i+1}}$. Then each map of a simple closed curve into

$$
K^{t_{i}} \cup K^{t_{i+1}} \cup\left(h(\operatorname{Bd} D) \cap \bigcup_{t_{i} \leq t \leq t_{i+1}} E_{t}\right)
$$

is homotopic to a map of the simple closed curve into $E_{t_{i}} \cap \operatorname{Int} S^{2} \cap U$.

We now prove the following: given any map

$$
d: \operatorname{Bd} D \rightarrow E_{t}^{2} \cap \operatorname{Int} S^{2} \cap U
$$

there exists a map

$$
\text { d: } D \rightarrow E_{t}^{2} \cap \operatorname{Int} S^{2} \cap U
$$

such that $d$ is an extension of $d$.

Recall that $E_{\imath} \cap S^{2}=J_{t}$, a simple closed curve. Let $J_{\mathfrak{t}}^{\prime}$ be a simple closed curve in Int $J_{t}$ on $E_{t}$ such that

(i) $d(\mathrm{Bd} D) \subset$ Int $J_{t}^{\prime}$

(ii) $J_{i}^{\prime} \cap U$ consists of a finite number of open $\operatorname{arcs} B_{1}, \cdots, B_{i}$. Since $U \cap E_{\imath}$ is a disk, the map $d$ admits an extension $d^{\prime}: D \rightarrow U \cap E_{t}$. Let $C$ be the component of $D-\left(d^{\prime}\right)^{-1}\left(d^{\prime}(D) \cap J_{i}^{\prime}\right)$ containing $\operatorname{Bd} D$. Let $F$ be a component of the boundary of $C$ relative to $D$. The set $d^{\prime}(F)$ must lie in one of the $\operatorname{arcs} B_{j}$. Therefore $\left.d^{\prime}\right|_{c}$ can be extended to map $F$ and the components in Int $D$ of $D-F$ into $B_{j}$. In this way, we extend $\left.d^{\prime}\right|_{c}$ to all of $D$ and obtain the required extension

$$
\text { d: } D \rightarrow U \cap \mathrm{Cl}\left(\operatorname{Int} J_{t}^{\prime}\right) \subset U \cap E_{t} \cap \operatorname{Int} S^{2} .
$$

Thus we have shown the following: each map of a simple closed curve into

$$
K^{i} \cup K^{t_{i+1}} \cup\left(h(\mathrm{Bd} D) \cap \bigcup_{t_{i} \leq t \leq t_{i+1}} E_{t}\right)
$$

is homotopic in $U \cap$ Int $S^{2}$ to a constant map.

(C) Now consider the disjoint connected sets $A_{j}^{t}$ in $D$ each of 
which contains at least two points of $\mathrm{Bd} D$. Let $O_{j}^{4},\left(i=t_{1}, \cdots, t_{n}\right.$; $\left.j=1, \cdots, k\left(t_{n}\right)\right)$ be a collection of disjoint open subsets of $D$ such that $A_{j}^{i} \subset O_{j}^{i}$.

In each $O_{j}^{t}$ choose a point $p \in O_{j}^{i} \cap$ Int $D$. For each $q \in A_{1}^{i} \cap \mathrm{Bd} D$ we take an arc $[p q]$ in $O_{j}^{q}$ such that $[p q] \cap\left[p q^{\prime}\right]=p$ for $q^{\prime} \neq q$ and $q, q^{\prime} \in A_{j}^{i} \cap \mathrm{Bd} D$. Assume also that $[p q] \cap \mathrm{Bd} D=q$. Let $L_{j}^{i}$ be the finite graph (a "spoke" with $p$ as center) in $O_{j}^{i}$ formed by the union of all such arcs in $O_{j}^{q}$.

(D) Let $L^{t}=L_{1}^{t} \cup \ldots \cup L_{k(t)}^{t}$ for $t=t_{1}, \cdots, t_{n}$. Consider a component $M$ of $D-U_{i=1}^{n} L^{t_{i}}$. The closure of $M$ is a topological disk. We claim that the boundary of $M$ in $D$ must lie in $L^{t_{i}} \cup L^{t_{i+1}}$ for some $1 \leqq i \leqq n-1$. Consider $t_{a}, t_{b}, t_{c}$ such that $1 \leqq a<b<c \leqq n$. Then $h^{-1}\left(h(D) \cap E_{t_{b}}\right)$ separates any $A_{i}^{l}\left(i=1, \cdots, k\left(l_{a}\right)\right)$ from any $A_{j}^{l_{c}}$, $\left(j=1, \cdots, k\left(t_{c}\right)\right)$.

Now fix $i$ and $j$. Since $A_{i}^{t_{a}}$ and $A_{j}^{t_{c}}$ are connected sets and contain points of $\mathrm{Bd} D$ some component of $h^{-1}\left(h(D) \cap E_{t_{t}}\right)$ containing at least two points of $\mathrm{Bd} D$ must separate $A_{i}^{t_{a}}$ from $A_{f}^{t_{f}}$. But such a component is one of the sets $A_{m}^{t_{b}},\left(1 \leqq m \leqq k\left(t_{b}\right)\right)$. Therefore $L_{m}^{t^{3}}$ separates $L_{i}^{b^{a}}$ from $L_{f}^{\boldsymbol{b}_{c}}$ in $D$ and our claim is verified.

We now extend $f$ to a map $f_{1}$ such that

$$
f_{1}: L_{i}^{t} \rightarrow K_{i}^{t} ; \quad\left(t=t_{1}, \cdots, t_{n} ; i=1, \cdots, k\left(t_{n}\right)\right) .
$$

Again let $M$ be a component of $D-U_{i=1} L^{i}$. Then

$$
f_{1}(\mathrm{Bd} M) \subset K^{t_{i}} \cup K^{t_{i+1}} \cup\left(f(\mathrm{Bd} D) \cap \bigcup_{t_{i} \leq t t_{i+1}} E_{t}\right)
$$

for some $i$. Thus, by part (B), $f_{1}$ can be extended to map $M$ into $U$ Int $S^{2}$. By extending $f_{1}$ over all such components $M$ we obtain a map

$$
g: D \rightarrow U \cap \operatorname{Int} S^{2}
$$

such that

$$
\left.g\right|_{\mathrm{Bd} D}=f \text { on } \mathrm{Bd} D .
$$

This completes the proof that $S^{2}-\left\{p_{a}, p_{b}\right\}$ is locally tame.

(E). We finish the proof of the theorem by showing that $S^{2}$ can be homeomorphically approximated by 2 -spheres from either complementary domain and is therefore tame, [4]. Let $\epsilon>0$ be given. We first consider the problem of approximating from the exterior of $S^{2}$. Choose $U$ to be a round open ball about $p_{b}$ of diameter less than $\epsilon / 2$. Choose $t^{\prime}$ such that $J_{t} \subset U$ for $t^{\prime} \leqq t<b$. On $E_{t^{\prime}}$, the simple closed curves $J_{t}$, and $B d U \cap E_{t^{\prime}}$ bound an annulus $A$. We now discard that 
part of $S^{2}$ lying above $E_{t^{\prime}}$ and add to remainder of $S^{2}$ the annulus $A$ plus the portion of $\mathrm{Bd} U$ lying above $E_{t^{\prime}}$. By performing a similar modification about $p_{a}$ we obtain a 2 -sphere $\hat{S}^{2}$ such that

$$
\left\{p_{a}, p_{b}\right\} \subset \text { Int } \hat{S}^{2} \text { and } S^{2} \subset \mathrm{Cl}\left(\operatorname{Int} \hat{S}^{2}\right) \text {. }
$$

Further $\hat{S}^{2}$ is tame as it is locally tame modulo two tame simple closed curves. Thus by pushing $\hat{S}^{2}$ slightly into its exterior we obtain the desired $\epsilon$-approximation.

The interior approximation is even easier. We choose curves $J_{t_{1}}$ and $J_{t_{2}}$ sufficiently close to $p_{a}$ and $p_{b}$ respectively, throw away the parts of $S^{2}$ lying below $E_{t_{1}}$ and above $E_{t_{2}}$, add Int $J_{t_{1}}$ and Int $J_{t_{2}}$ and then push our modified tame sphere slightly into its interior.

\section{REFERENCES}

1. J. W. Alexander, On the subdivision of 3-space by a polyhedron, Proc. Nat. Acad. Sci. U.S.A. 10 (1924), 6-8.

2. R. H. Bing, A surface is tame if its complement is 1-ULC, Trans. Amer. Math. Soc. 101 (1961), 294-304.

3. - Spheres in $E^{3}$, Amer. Math. Monthly 71 (1964), 353-364.

4. - Conditions under which a surface in $E^{3}$ is tame, Fund. Math. 47 (1959), $105-139$.

5. W. Greub, Die semilinearen Abbildungen, S.-B. Heidelberger Akad. Wiss. Math.-Nat. Kl. 1950, 205-272.

6. E. E. Moise, Affine structures in 3-manifolds. II: Positional properties of 2spheres, Ann. of Math. (2) 55 (1952), 172-176.

University of Washington, Seattle, Washington 98105 